\title{
Leeches alleviates diabetic macroangiopathy by increasing nitric oxide production in rat aorta and restoring endothelium-dependent vasodilatation
}

\section{Type}

Research paper

\section{Keywords}

NO, endothelium, diabetic macroangiopathy, aorta, Leeches

\begin{abstract}
Introduction

Diabetes mellitus (DM) is a chronic metabolic disorder characterized by elevated blood glucose level over a prolonged period, leading to severe damage in tissues including the heart, blood vessels, eyes and the kidneys. Danzhi Jiangtang Capsule (DJC) is an effective drug for diabetes, but the mechanism responsible for its efficacy remains unknown. This study aimed to explore the effective ingredient of DJC that ameliorated diabetes and the possible mechanisms.
\end{abstract}

\section{Material and methods}

We orally treated streptozotocin (STZ)-induced diabetic rats with $540 \mathrm{mg} / \mathrm{kg} \mathrm{DIC}$ or the same dose of its four active components, namely Leeches, Pseudostellaria Polysaccharides (PP), Paeonia Suffruticosa Andr (PSA) and Rehmannia Glutinosa Libosch (RGL), respectively for 8 weeks.

\section{Results}

Although all of these components could reduce blood glucose levels in diabetic rats, the extent of alleviation of DJC was more pronounced than all of its four ingredients. Unlike the other three components, Leeches is the only effective ingredient of DJC that decreased tetrahydrobiopterin (BH4) oxidation to activate endothelial nitric oxide synthase (eNOS), and increased nitric oxide (NO) production, leading to improved endothelium-dependent relaxation both in diabetic rats and in immortalized human mesangial cells under the stimulation of high glucose.

\section{Conclusions}

Leeches could alleviate diabetic macroangiopathy by inhibiting NO release in endothelial cells under high-glucose condition. 


\title{
Leeches alleviates diabetic macroangiopathy by increasing nitric oxide
}

production in rat aorta and restoring endothelium-dependent vasodilatation

\author{
Zhaohui Fang ${ }^{1, *}$, Xiu $\mathrm{Hu}^{2}$, Zhi Chen ${ }^{3}$, Jing $\mathrm{Xie}^{2}$, Di Wu ${ }^{2}$, Yundong Yin ${ }^{2}$ \\ ${ }^{1}$ Department of Endocrine, the First Hospital Affiliated to Anhui University of \\ Traditional Chinese Medicine; Anhui Provincial Academy of Science of Traditional \\ Chinese Medicine, No. 103 Anmeishan Road, Shushan District, Hefei 230038, Anhui, \\ China \\ ${ }^{2}$ Graduate Institute, Anhui University of Chinese Medicine, No.103 Meishan Road, \\ Hefei 230038, Anhui, China \\ ${ }^{3}$ Department of Endocrine, Taizhou Hospital of Traditional Chinese Medicine, No. 86 \\ Jichuandong Road, Taizhou 225300, China
}

*Corresponding author

Zhaohui Fang

Department of Endocrine, the First Hospital Affiliated to Anhui University of Traditional Chinese Medicine; Anhui Provincial Academy of Science of Traditional Chinese Medicine, No. 103 Anmeishan Road, Shushan District, Hefei 230038, Anhui, China

Email: fangzhaohui1111@163.com

Tel: 86-13965059097

Running title: Leeches alleviates diabetic macroangiopathy 


\begin{abstract}
Introduction: Diabetes mellitus (DM) is a chronic metabolic disorder characterized by elevated blood glucose level over a prolonged period, leading to severe damage in tissues including the heart, blood vessels, eyes and the kidneys. Danzhi Jiangtang Capsule (DJC) is an effective drug for diabetes, but the mechanism responsible for its efficacy remains unknown. This study aimed to explore the effective ingredient of DJC that ameliorated diabetes and the possible mechanisms.
\end{abstract}

Materials and methods: We orally treated streptozotocin (STZ)-induced diabetic rats with $540 \mathrm{mg} / \mathrm{kg}$ DIC or the same dose of its four active components, namely Leeches, Pseudostellaria Polysaccharides (PP), Paeonia Suffruticosa Andr (PSA) and Rehmannia Glutinosa Libosch (RGL), respectively for 8 weeks.

Results: Although all of these components could reduce blood glucose levels in diabetic rats, the extent of alleviation of DJC was more pronounced than all of its four ingredients. Unlike the other three components, Leeches is the only effective ingredient of DJC that decreased tetrahydrobiopterin $\left(\mathrm{BH}_{4}\right)$ oxidation to activate endothelial nitric oxide synthase (eNOS), and increased nitric oxide (NO) production, leading to improved endothelium-dependent relaxation both in diabetic rats and in immortalized human mesangial cells under the stimulation of high glucose.

Conclusion: Leeches could alleviate diabetic macroangiopathy by inhibiting NO release in endothelial cells under high-glucose condition.

Keyword: Leeches; diabetic macroangiopathy; NO; endothelium; aorta 


\section{Introduction}

Diabetes mellitus (DM) is a chronic metabolic disorder characterized by elevated blood glucose level over a prolonged period, leading to severe damage in tissues including the heart, blood vessels, eyes and the kidneys. Among all the complications, diabetic macroangiopathy is the most prevalent and highly lethal one, which accounts for 70\%$80 \%$ of deaths in all diabetic patients. The prevalence of atherosclerosis is higher in diabetic individuals than in non- diabetic patients, commonly characterized as with younger onset age, faster progression, more severe condition and higher mortality rate. Meanwhile, diabetic complication such as coronary heart disease, myocardial infarction and acute cerebrovascular disease is the leading cause of death in diabetes [1]. Diabetic macroangiopathy is characterized as early and severe atherosclerosis and resultant blood clot accumulated in large vessels including aorta, coronary arteries, basilar arteries, renal arteries and peripheral arteries, due to excessive or abnormal neovasculogenesis and angiogenesis [2]. Therefore, increasing studies have been conducted to prevent diabetic macroangiopathy [3].

Endothelial cells in the blood vessels play an important role in controlling the automatic regulation of contractility and vascular homeostasis in vascular smooth muscle cells by releasing a variety of vasoconstrictor-secreted factors, such as thromboxane, prostacyclin and nitric oxide (NO) [4, 5]. Insufficient synthesis of NO is one of the causes of vascular and endothelium dysfunctions in diabetic condition [5]. Therefore, nitric oxide synthase (NOS), which produces NO, is also involved in the process of diabetes-induced vascular dysfunction $[6,7]$.

Danzhi Jiangtang Capsule (DJC), as one Traditional Chinese Medicine, is an effective drug for diabetes in rats [3], and it has been widely used in the clinic for a long period. Previous report demonstrated that DJC had a therapeutic effect in ameliorating high-fat 
diet-caused vascular damage. However, its therapeutic role in diabetic macroangiopathy has not been discovered yet. Since DJC has four active ingredients, all of which are also Traditional Chinese Medicine, namely Leeches, Pseudostellaria Polysaccharides (PP), Paeonia Suffruticosa Andr (PSA) and Rehmannia Glutinosa Libosch (RGL), we aimed to elucidate the key component(s) with anti-diabetic property and the mechanisms responsible in treating diabetic macroangiopathy. Therefore, this study explored the potential role and the mechanism of DJC, as well as its active ingredients, in diabetic macroangiopathy.

\section{Methods \& Materials}

\section{Animal model}

DJC is a mixture of 6 components, including Radix pseudostellariae, Rehmannia glutinosa Libosch, Rehmannia glutinosa Libosch, Alisma, tu Sizi, Leeches in the ratio of 5:4:4:3:2:5. The first 5 components were boiled in water for 2-3 times and 1-2 hours each time, then the combined decoctions were filtrated, concentrated and spray dried into powder. Leeches were dried, grounded into powder, mixed well with the powder of the other five components, and filled into capsules.

For evaluating the effective component of DJC, we selected four major active components in our study: Leeches, PP, PSA and RGL. Leeches were weighed after being dried and then ground into powder, while the other 3 were weighed after being dried and then boiled in water.

56 Sprague-Dawley male rats were randomly and equally divided into 7 groups, with 8 rats in each group. One group was intraperitoneally injected and orally gavaged with saline as control, and the other six groups were intraperitoneally injected with a singledose of streptozotocin (STZ, $50 \mathrm{mg} / \mathrm{kg}$, Sigma) to induce diabetes. Diabetic rats with 
high fasting blood glucose were orally treated with saline (DM group), DJC (DM+DJC), Leeches (DM + Leeches), PP (DM + PP), PSA (DM + PSA) and RGL (DM + RGL), respectively. All the drugs were orally administrated at a dose of $540 \mathrm{mg} / \mathrm{kg}$ for 8 weeks, and fasting blood-glucose level and blood pressure were measured as described before [8].

Each individual rat was placed in a transparent airtight chamber, which was flushed with $\mathrm{CO}_{2}$ gas that rapidly killed the rat. The chamber was flushed with air before being used again.

All studies were approved by the ethics commitment of the First Hospital Affiliated to Anhui University of Traditional Chinese Medicine. This study was performed in strict accordance with the NIH guidelines for the care and use of laboratory animals $(\mathrm{NIH}$ Publication No. 85-23 Rev. 1985).

\section{Isometric tension vasomotor studies}

Aortic vasomotor function was measured as described before [9]. Briefly, two rings with $2 \mathrm{~mm}$ length were collected from each thoracic aorta and mounted in organ bath chambers (Multi-Myograph 610M; Danish Myo Technology, Aarhus, Denmark) in Krebs-Henseleit buffer. Rings were constricted with $60 \mathrm{mM} \mathrm{KCl}$, typically achieving a contraction of 5 to $7 \mathrm{mN}$ of active tension after $5 \mathrm{~min}$ as the corrective value. After washing three times, rings were equilibrated for $30 \mathrm{mins}$, and then precontracted to approximately $50 \%$ of maximal tension with phenylephrine (typically $3 \times 10^{-6} \mathrm{~mol} / \mathrm{l}$ ). Acetylcholine was used to stimulate endothelial NO release to relax endotheliumdependent aorta. Endogenous NOS activity was inhibited by $10^{-4} \mathrm{~mol} / \mathrm{l} \mathrm{L}-\mathrm{N}(\mathrm{G})-$ Nitroarginine methyl ester (I-NAME) and sodium nitroprusside, which is a NO donor, which was used to measure endothelium-independent relaxation, expressed as a percentage of the phenylephrine precontraction. 


\section{Measurement of nitrite and nitrate production}

Rat aorta from different groups were cut into pieces and cultured in the medium for 24 hours at $37^{\circ} \mathrm{C}$, and then supernatant was used for analyzing the production of $\mathrm{NO}^{-}$. After adding samples or serial diluted nitrate standard with vanadium (III) chloride into 96-well plate, Griess reagents were also loaded to the wells followed with sulphanilamide and N-1-Naphthylethylenediamine dihydrochloride. Diluting medium was used as blank. Nitrite production was measured in an identical way, except for that samples or nitrite standards were only exposed to Griess reagents. After incubation for 30 to $45 \mathrm{mins}$, plates were read at the absorbance of $540 \mathrm{~nm}$ [10].

\section{Western blot}

Western blot was performed as previously described [11, 12]. Briefly, aorta was collected from different groups, and homogenized in the lysis buffer with protease inhibitors. After centrifuged at 12,000 $\mathrm{g}$ for $30 \mathrm{mins}$, pellet was homogenized in TrisEDTA buffer, and then centrifuged again at 100,000 $g$ for 20 mins. Total protein concentration in all fractions was determined by bicinchoninic acid kit. Denatured proteins were separated in sodium dodecyl sulfate gel and transferred into membrane, followed by incubation with endothelial nitric oxide synthase (eNOS) primary antibody (1:800 for membrane fractions, 1:500 for cytosol) overnight after blocking in 5\% milk to detect monomer and total eNOS. To analyze eNOS dimer expression, undenatured proteins were separated by cold electrophoresis at $4{ }^{\circ} \mathrm{C}$ with detection and visualization as described above. All the antibodies were purchased from Cell Signaling Technology.

\section{Measurement of tetrahydrobiopterin $\left(\mathrm{BH}_{4}\right)$ and total biopterin}

$\mathrm{BH}_{4}$ and total biopterin were determined according to a previously described method $[13,14]$. Aorta was lysed in the extraction buffer, and urine was collected in the tube. All the samples were injected into a ultraperformance liquid chromatography system. 
$\mathrm{BH}_{4}$ concentration $=$ total biopterin $-\left(\mathrm{BH}_{2}+\right.$ biopterin $)$. The percentage of $\mathrm{BH}_{4}$ oxidation $=100-\left(\mathrm{BH}_{4} \times 100 /\right.$ biopterin $)$.

\section{Diamino-fluorescein detection of $\mathrm{NO}$}

Immortalized human mesangial cells (ihMCs) (ATCC) were cultured in Dulbecco's modified Eagle's medium (DMEM, Gibco, Grand Island, NY, USA) with the addition of $10 \%$ fetal bovine serum (FBS, Gibco). After incubation with high glucose in the presence or absence of Leeches for 24 hours, ihMCs were incubated with $10 \mu \mathrm{M}$ DAFFM diacetate (Invitrogen, Carlsbad, CA, USA) for 30 mins, which is a NO-sensitive fluorescent dye, and washed with PBS. Then ihMCs were observed using a fluorescence microscope. The intensity of fluorescence was calculated by ImageJ software.

\section{Statistical analysis}

All the data were expressed as mean \pm SEM. One or two-way ANOVA analysis with a Tukey's post hoc test was used to compare data from different groups. All the analysis was performed using GraphPad Prism software. $p$ value $<0.05$ was considered as significant difference.

\section{Results}

\section{Leeches improved endothelium-dependent relaxation of the aortic vessel in diabetic}

rats

After a single-dose injection of STZ, rats were induced with diabetes as evidenced by increased fasting blood-glucose level compared with the normal group (18.5 \pm 2.3 v.s. $8.6 \pm 0.4 \mathrm{mmol} / \mathrm{L})$ (Figure 1a).

DJC, as an effective anti-diabetic drug in the clinic, significantly decreased blood glucose level in diabetic rats, and its 4 active ingredients, including Leeches, PP, PSA 
and RGL, also reduced blood glucose level after the 8-week treatment. The effective dose of DJC was $270-1080 \mathrm{mg} / \mathrm{kg}$ in rats (data not shown). Here, we used the same dose of each component and DJC (540 mg/kg). Diabetic rats displayed increased acetylcholine-induced endothelium-dependent relaxation compared to control rats, which could only be restored by Leeches and DJC. Acetylcholine is the agonist of endothelial eNOS, which facilitates eNOS to release NO from endothelial cells. While the other three ingredients, including PP, PSA and RGL, failed to reduce aortic vasodilation (Figure 1b). Furthermore, Leeches improved endothelium-dependent relaxation in response to different doses of acetylcholine $\left(10^{-9}\right.$ to $\left.10^{-5} \mathrm{~mol} / \mathrm{L}\right)$ (Figure 1c). Sodium nitroprusside, as the direct NO donor, was used to evaluate endotheliumindependent aortic relaxation, due to its stimulation on smooth muscle relaxation to exogenous nitric oxide. But Leeches showed no effect on sodium nitroprusside (Figure 1d). All these data suggested that all the four active ingredients of DJC could effectively lower blood glucose level in diabetic rats, but Leeches was the only active monomer of DJC that remarkably improved endothelium-dependent relaxation.

Leeches restored $\mathrm{NO}_{x}^{-}$level and $\mathrm{eNOS}$ dimer-to-monomer ratio in the aortic vessel of diabetic rats

Since NO is an important cellular signaling molecule that modulates vascular tone and relaxation, we speculated that NO participated in the process of Leeches-improved vascular relaxation in diabetic rats. Both nitrate $\left(\mathrm{NO}_{3}\right)$ and nitrite $\left(\mathrm{NO}_{2}\right)$ are stable $\mathrm{NO}$ end products, and as expected, ex vivo Griess reaction indicated that NOx- production was elevated by in the aorta of diabetic rats, which was restored after Leeches treatment (Figure 2a). Nitric oxide synthases (NOSs) are a family of enzymes catalyzing the production of NO from L-arginine. There are three isoforms of NOS, including eNOS, neuronal NOS (nNOS) and inducible NOS (iNOS). Leeches recovered endothelium- 
dependent, but not endothelium-independent, relaxation, indicating that NO ameliorated by Leeches was derived from eNOS. Subsequently, we observed that there was no difference in the eNOS expression between normal and diabetic rats with or without Leeches (Figure 2b and 2c). Of note, the eNOS dimer and monomer expression was altered in the opposite manners in different groups (Figure 2b). The eNOS dimer/monomer ratio, which was downregulated in diabetic rats, was augmented after Leeches administration (Figure $2 \mathrm{~b}$ and $2 \mathrm{~d}$ ). As dimer eNOS is considered as the active form, Leeches therefore improved eNOS activity as evidenced by the increased ratio of eNOS dimer/monomer. These data suggested that Leeches improved aortic vasodilatation in diabetic rats by increasing eNOS activity without altering eNOS expression.

\section{Leeches restored $\mathrm{BH}_{4}$ level in the aortic vessel of diabetic rats}

$\mathrm{BH}_{4}$ is an essential cofactor of eNOS to produce NO by participating in the enzymatic process of L-arginine oxidation. Because $\mathrm{BH}_{4}$ is one of the biopterin derivatives, we evaluated both the $\mathrm{BH}_{4}$ and total biopterin expression levels in the aorta and urine from different groups. Not surprisingly, the expression levels of $\mathrm{BH}_{4}$ and total biopterin were remarkably reduced in both the aorta and urine of diabetic rats, which could be restored by Leeches treatment (Figure $3 \mathrm{a}$ and $3 \mathrm{c}$ ). Correspondingly, the ratio of $\mathrm{BH}_{4} /$ total biopterin was also significantly decreased in diabetic rats, and restored by Leeches in the aorta and urine of diabetic rats (Figure $3 b$ and $3 d$ ). On the contrary, diabetes increased the percentage of $\mathrm{BH}_{4}$ oxidation in the aorta and urine, and after Leeches treatment, $\mathrm{BH}_{4}$ oxidation declined (Figure $3 \mathrm{e}$ and $3 \mathrm{f}$ ). These results indicated that, with the increased oxidation of $\mathrm{BH}_{4}$ as a result of diabetes, eNOS gradually lost its function, while Leeches could recover $\mathrm{BH}_{4}$ oxidation and eNOS activity. Taken together, Leeches could ameliorate eNOS activity in the aortic endothelium which were impaired 
by diabetes.

\section{Leeches restored NO level in ihMCs cultured in high glucose medium}

Next, we used ihMCs cultured in high glucose DMEM to mimic high blood glucose level in vivo. NO production was significantly reduced in the medium with high glucose (HG, $30 \mathrm{mM})$ compared to normal glucose $(5.5 \mathrm{mM})$, and was restored in response to Leeches supplement in HG medium (Figure $4 \mathrm{a}$ and $4 \mathrm{~b}$ ). eNOS expression was not changed in either normal or HG medium, with or without Leeches (Figure 4c and 4d). Moreover, the radio of dimer/monomer eNOS was reduced in HG medium and reversed with the supplement of Leeches (Figure $4 \mathrm{c}$ and $4 \mathrm{e}$ ). Hence, high glucose could downregulate NO production by decreasing eNOS activity in ihMCs, which could be restored by Leeches supplement.

\section{Leeches restored $\mathrm{BH}_{4}$ level in ihMCs cultured in high glucose medium.}

Next, we also evaluated $\mathrm{BH}_{4}$ and total biopterin levels in ihMCs. $\mathrm{BH}_{4}$, total biopterin expression and the ratio of $\mathrm{BH}_{4} /$ total biopterin were all reduced in $\mathrm{HG}$ medium and recovered by Leeches supplement (Figure 5a and 5b). Furthermore, Leeches could reduce $\mathrm{BH}_{4}$ oxidation which was elevated in $\mathrm{HG}$ medium (Figure 5c).

\section{Discussion}

Our study demonstrated that Leeches, as well as DJC, alleviated diabetic macroangiopathy by releasing eNOS-derived NO to improve aortic relaxation. This study is the first instance to discover that Leeches is the only active ingredient in DJC to both relax vasodilatation and decrease blood glucose level in diabetic rats.

DJC, as a Traditional Chinese Medicine combined with conventional diabetes treatment, has been widely used to treat diabetes in the clinic for a long period of time $[15,16]$. It could effectively reduce blood glucose level in diabetic patients $[17,18]$ and ameliorate 
some diabetic complications, such as kidney dysfunction, macroangiopathy and myocardial fibrosis $[7,18,19]$. A variety of mechanisms is related to the protective roles of DJC in diabetes. DJC increased insulin sensitivity in both type 1 and type 2 diabetic rat models by improving beta cell function, suppressing beta cell apoptosis and ameliorating beta cell oxidation $[3,20,21]$. Meta-analysis suggested that the combination of DJC and conventional treatment effectively reduced insulin resistance in diabetic patients [22]. Moreover, the combination of DJC and insulin aspart 30 protected against type 2 diabetic nephropathy through controlling blood glucose, urinary albumin excretion, serum homocysteine and Cys-C levels [23]. Another study demonstrated that the regulation of transforming growth factor-beta $1 /$ Smads signaling pathway, as well as its downstream connective tissue growth factor expression, was involved in the alleviated kidney damage and improved diabetic symptoms in diabetic rats fed on DJC [12].

Our study evaluated the anti-diabetic property of DJC, as well as its active ingredients, to treat diabetes and its complications, such as diabetic macroangiopathy. Previous study discovered that DJC had protective and therapeutic effects against diabetic macroangiopathy in type 2 diabetic rats, which might involve the repaired islet cells and endothelial cells by reducing the expression of Plasminogen Activator Inhibitor, Endothelin-1 and Thromboxane A2 and inducing the production of NO and Prostaglandin I2. However, there are four active ingredients in DJC, including Leeches, PP, PSA and RGL. Although earlier studies indicated that PP, PSA and RGL might have potential roles in diabetic rats [24-28]. The underlying mechanisms are still unclear.

Our study for the first time demonstrated that all these four monomers could partially improve blood glucose level in STZ-induced diabetic rats without altering systolic 
blood pressure. Unexpectedly, unlike the other three components, Leeches is the only effective component of DJC to relax vasodilatation. Considering that so far there is no research on Leeches and diabetes, our study is the first report discovering the relationship between Leeches and diabetes, where Leeches was shown to effectively attenuate STZ-induced diabetes in rats. Furthermore, Leeches ameliorated endothelium-dependent aortic relaxation, to a similar extent as DJC, which implied that Leeches could serve as a promising therapeutic drug to treat diabetes and its vasculumrelated complications, such as diabetic macroangiopathy, in diabetic patients.

Macroangiopathy is one of the most common complications in diabetic patients, and currently the major cause of morbidity and mortality in patients with type 1 and type 2 diabetes. Compared to non-diabetic individuals, diabetic patients are more prone to atherosclerosis that can be attributed to epigenetic factors [29], which also develops rapidly to coronary heart disease, cerebrovascular accidents and gangrene of lower limbs. According to previous reports, $20 \%$ of patients with peripheral vascular diseases were found to have diabetes. Currently, it is believed that the occurrence of diabetic macroangiopathy is related to the age of patients, the progress and control of diabetes. A variety of pathogenesis is involved in the development of diabetic macroangiopathy, including hyperinsulinemia/insulin resistance, lipid metabolism disorder, aortic endothelium dysfunction, abnormal blood coagulation, hyperglycemia, hormonal regulation disorder and genetic factors. Among all these mechanisms, endothelium dysfunction, as well as its loss on the modulatory role, may be an important factor to initiate the process of diabetic vascular diseases, which could be improved by Leeches treatment. Because endothelium controls the tone of vascular smooth muscle cells by secreting vasodilator factors such as NO.

NO is catalyzed from NOS, which has three different isoforms, eNOS, iNOS which is 
involved in immune responses, and nNOS which only exists in neuronal cells. Since vasodilation impaired by diabetes is endothelium-dependent, eNOS is the only isoform that is involved in this process. Our results showed that eNOS activity was improved after Leeches treatment with no alteration of eNOS expression in diabetic rats, therefore, as the requirement of the redox-sensitive co-factor, $\mathrm{BH}_{4}$ is critical in this enzymatic process. However, the role of $\mathrm{BH}_{4}$ in diabetes-induced macroangiopathy remains unknown. Our study demonstrated that $\mathrm{BH}_{4}$ oxidation, which is related to eNOS activity, was also involved in the development of diabetic macroangiopathy.

Taken together, DJC, as well as its four active ingredients, could effectively improve blood glucose level with no alteration in blood pressure in diabetic rats. However, Leeches is the only component of DJC that improved endothelium-dependent aortic relaxation. Leeches recovered eNOS-derived $\mathrm{NO}$ production by improving $\mathrm{BH}_{4}$ oxidation in both diabetic rats and cultured ihMCs. Hence, Leeches could serve as a potential therapeutic drug to treat diabetic macroangiopathy by attenuating endothelium dysfunction and improving aortic vasodilatation.

\section{Funding}

None.

\section{Disclosure of potential conflicts of interest}

The authors declare that they have no competing interests.

\section{Acknowledgements}

Not applicable. 


\section{Reference}

1. Getz GS. Report on the workshop on diabetes and mechanisms of atherogenesis. September 17th and 18th, 1992, Bethesda, Maryland. Arteriosclerosis and Thrombosis $1993 ; 13: 459-64$

2. Madonna R, Pieragostino D, Balistreri CR, et al. Diabetic macroangiopathy: Pathogenetic insights and novel therapeutic approaches with focus on high glucosemediated vascular damage. Vascular Pharmacology 2018.

3. Zheng S, Zhao M, Wu Y, Wang Z and Ren Y. Suppression of pancreatic beta cell apoptosis by Danzhi Jiangtang capsule contributes to the attenuation of type 1 diabetes in rats. BMC Complementary and Alternative Medicine 2016; 16:31.

4. Cacanyiova S, Dovinova I and Kristek F. The role of oxidative stress in acetylcholine-induced relaxation of endothelium-denuded arteries. Journal of Physiology and Pharmacology 2013; 64:241-7.

5. Durand MJ and Gutterman DD. Diversity in mechanisms of endotheliumdependent vasodilation in health and disease. Microcirculation 2013; 20:239-47.

6. Jarajapu YP, Cai J, Yan Y, et al. Protection of blood retinal barrier and systemic vasculature by insulin-like growth factor binding protein-3. PloS One 2012; 7:e39398. 7. Lu Y, Chen Y, Li R, et al. Protective effects of Danzhi jiangtang capsule on vascular endothelial damages induced by high-fat diet and palmitic acid. Biomedicine and Pharmacotherapy 2018; 107:1631-40.

8. Zaidun NH, Sahema ZCT, Mardiana AA, Santhana RL, Latiff AA and Syed Ahmad Fuad SB. Effects of naringenin on vascular changes in prolonged hyperglycaemia in fructose-STZ diabetic rat model. Drug Discoveries \& Therapeutics $2019 ; 13: 212-21$.

9. Paredes-Carbajal MC, Monsalvo I, Hernández-Díaz C, Regla I, Demare P and 
Mascher D. Effects of ranolazine on vasomotor responses of rat aortic rings. Archives of Medical Research 2013; 44:8-12.

10. Miranda KM, Espey MG and Wink DA. A rapid, simple spectrophotometric method for simultaneous detection of nitrate and nitrite. Nitric Oxide 2001; 5:62-71.

11. Komers R, Schutzer WE, Reed JF, et al. Altered endothelial nitric oxide synthase targeting and conformation and caveolin-1 expression in the diabetic kidney. Diabetes 2006; 55:1651-9.

12. Liu Z, Luo H, Zhang L, et al. Hyperhomocysteinemia exaggerates adventitial inflammation and angiotensin II-induced abdominal aortic aneurysm in mice. Circulation Research 2012; 111:1261-73.

13. Faria AM, Papadimitriou A, Silva KC, Lopes de Faria JM and Lopes de Faria JB. Uncoupling endothelial nitric oxide synthase is ameliorated by green tea in experimental diabetes by re-establishing tetrahydrobiopterin levels. Diabetes 2012; 61:1838-47.

14. Fekkes D and Voskuilen-Kooijman A. Quantitation of total biopterin and tetrahydrobiopterin in plasma. Clinical Biochemistry 2007; 40:411-3.

15. Fang Z and Ni Y. Intervention effect of Danzhijiangtang Capsule on vascular endothelium injury induced by oxidative stress in type two diabetes mellitus. Chinese Journal of Clinical Rational Drug Use 2011; 4:27-8.

16. Shu Y, Fang Z and Bao T. Therapeutic effects of Danzhijiangtang Capsule on patients with type two diabetes mellitus. Journal of Emergency in Traditional Chinese Medicine 2007; 16:158-9.

17. Fang Z, Hu X, Chen Z, et al. Radix pseudostellariae of Danzhi Jiangtang capsule relieves oxidative stress of vascular endothelium in diabetic macroangiopathy. Saudi Pharm J 2020; 28:683-91. 
18. Sun M, Bu W, Li Y, et al. Danzhi Jiangtang Capsule ameliorates kidney injury via inhibition of the JAK-STAT signaling pathway and increased antioxidant capacity in STZ-induced diabetic nephropathy rats. Bioscience Trends 2019; 12:595-604.

19. Zheng SG, Tao SJ, Zhao MQ, Ren YN and Wu YJ. [Effect of Danzhi Jiangtang Capsule on Myocardial Fibrosis in Diabetic Rats]. Zhong Yao Cai Journal of Chinese Medicinal Materials 2015; 38:2120-4.

20. Wu YJ, Fang ZH, Zheng SG, Wu YB, Wang Z and Lu MA. [Effect of danzhi jiangtang capsule combined exercise on the protein expression of NADPH oxidase p22phox in pancreatic tissues of diabetic rats]. Zhongguo Zhong Xi Yi Jie He Za Zhi Zhongguo Zhongxiyi Jiehe Zazhi Chinese Journal of Integrated Traditional and Western Medicine 2013; 33:641-5.

21. Wu YJ, Fang ZH and Zheng SG. [Effects of danzhi jiangtang Capsule combined exercise on pancreatic oxidative stress and islet beta-cell function in diabetic rats]. Zhongguo Zhong Xi Yi Jie He Za Zhi Zhongguo Zhongxiyi Jiehe Zazhi Chinese Journal of Integrated Traditional and Western Medicine 2012; 32:1531-4.

22. Zhao J, Ding L, Bao T, et al. Meta-analysis of adjusting blood lipid of type 2 diabetes mellitus by Danzhi Jiangtang capsule. Chinese Archives of Traditional Chinese Medicine 2015; 33:570-3.

23. Jourdan T, Szanda G, Cinar R, et al. Developmental Role of Macrophage Cannabinoid-1 Receptor Signaling in Type 2 Diabetes. Diabetes 2017; 66:994-1007.

24. Zhao GH, Shen YS, Ma JB, Li F and Shi XQ. [Protection of polysaccharides$2 b$ from mudan cortex of Paeonia suffruticosa andr on diabetic cataract in rats]. Zhongguo Zhong Yao Za Zhi Zhongguo Zhongyao Zazhi China Journal of Chinese Materia Medica 2007; 32:2036-9.

25. Meng QY, Lv XF and Jin XD. [Effect of Rehmannia glutinosa Libosch water 
extraction on gene expression of proinsulin in type 2 diabetes mellitus rats]. Zhong Yao Cai Journal of Chinese Medicinal Materials 2008; 31:397-9.

26. Zhang RX, Jia ZP, Kong LY, et al. Stachyose extract from Rehmannia glutinosa Libosch. to lower plasma glucose in normal and diabetic rats by oral administration. Pharmazie 2004; 59:552-6.

27. Fang ZH, Duan XC, Zhao JD, Wu YJ and Liu MM. Novel Polysaccharide H-12 from Pseudostellaria Heterophylla Alleviates Type 2 Diabetes Mellitus. Cellular Physiology and Biochemistry 2018; 49:996-1006.

28. Hu J, Pang W, Chen J, Bai S, Zheng Z and Wu X. Hypoglycemic effect of polysaccharides with different molecular weight of Pseudostellaria heterophylla. BMC Complementary and Alternative Medicine 2013; 13:267.

29. Jia X, Cheng H and Xiao Y. Unfolding the pathogenesis of systemic sclerosis through epigenomics. STEMedicine 2020; 1:e5. 


\section{Figure Legends}

Figure 1. Leeches restored endothelial-dependent relaxation in the aorta of diabetic rats. (a) The blood glucose levels of rats in normal rats, diabetic rats with or without feeding drugs. Normal v.s. DM or DM+drugs, $* * * \mathrm{P}<0.001$; DM v.s. $\mathrm{DM}+$ drugs, \#P<0.001. (b) Endothelium-dependent relaxation of the aortic rings in response to $10^{-6} \mathrm{~mol} / \mathrm{L}$ acetylcholine in normal rats, diabetic rats, and diabetic rats fed Leeches. Normal v.s. DM, \#P<0.001; DM v.s. DM+drugs, $* \mathrm{P}<0.05 ; * * \mathrm{P}<0.01$. (c) Endothelial-dependent relaxation of the aortic rings in response to different concentrations of acetylcholine. $* * \mathrm{P}<0.01 ; * * * \mathrm{P}<0.001$. (d) Endothelium-independent relaxation of the aortic rings in response to different concentrations of nitroprusside. The data was represented as means \pm SD from three independent experiments.

Figure 2. Leeches restored $\mathrm{NO}_{\mathrm{x}}^{-}$levels and eNOS dimer-to-monomer ratio in the aorta of diabetic rats. (a) Nitrite $\left(\mathrm{NO}_{2}^{-}\right)$and nitrate $\left(\mathrm{NO}_{3}{ }^{-}\right)$, the stable $\mathrm{NO}$ end products, were quantified as a measurement of NO levels by Griess reaction in the aortic vessel of normal rats, diabetic rats, and diabetic rats fed Leeches. (b) Representative Western blots of eNOS dimer and monomer expression in the aorta of normal rats, diabetic rats, and diabetic rats fed on Leeches. (c) Densitometric analysis of the percentage of eNOSto-actin. (d) Densitometric analysis of the percentage of eNOS dimer-to-monomer. The data was represented as means $\pm \mathrm{SD}$ from three independent experiments. ${ }^{*} * \mathrm{P}<0.01$; $* * * \mathrm{P}<0.001$

Figure 3. Leeches restored $\mathrm{BH}_{4}$ levels in the aorta of diabetic rats. (a) $\mathrm{BH}_{4}$ and total biopterin expression in the aorta of normal rats, diabetic rats, and diabetic rats fed on Leeches. Results were corrected for the protein concentration and are expressed as $\mathrm{nmol} / \mathrm{mg}$ protein. (b) Ratio of $\mathrm{BH}_{4}$ and total biopterin expression in the aortic vessel of 
normal rats, diabetic rats, and diabetic rats fed Leeches. (c) $\mathrm{BH}_{4}$ and total biopterin expression in the urine of normal rats, diabetic rats, and diabetic rats fed Leeches. Results in the urine were corrected for $24 \mathrm{~h}$ urine volume. (d) Ratio of $\mathrm{BH}_{4}$ and total biopterin expression in the urine of normal rats, diabetic rats, and diabetic rats fed Leeches. (e-f) The percentage of $\mathrm{BH}_{4}$ to $\mathrm{BH}_{2}$ oxidation in the aorta $\mathrm{v}(\mathrm{e})$ and the urine (f). The data was represented as means \pm SD from three independent experiments. $* \mathrm{P}<0.05 ; * * \mathrm{P}<0.01 ; * * * \mathrm{P}<0.001$.

Figure 4. Leeches restored NO levels in ihMCs cultured with high glucose medium. (a) Representative photographs of DAF-FM indicating NO production. ihMCs were cultured for $24 \mathrm{~h}$ in normal glucose $(5.5 \mathrm{mmol} / \mathrm{L})$, high glucose $(\mathrm{HG})(30 \mathrm{mmol} / \mathrm{L})$, and HG with Leeches (100 mg/mL). (b) Quantification of the percentage of fluorescence units. (c) Western blots of eNOS, eNOS dimer and monomer expression in ihMCs. (d) Densitometric analysis of the percentage of eNOS-to-actin ratio. (e) Densitometric analysis of the percentage of eNOS dimer-to-monomer ratio. The data was represented as means $\pm \mathrm{SD}$ from three independent experiments. ${ }^{*} * \mathrm{P}<0.01 ; * * * \mathrm{P}<0.001$

Figure 5. Leeches restored $\mathrm{BH}_{4}$ level in ihMCs cultured with high glucose medium.

(a) Total biopterin and $\mathrm{BH}_{4}$ expression in ihMCs lysate. Results were corrected for the protein concentration and expressed as pmol/mg protein. (b) Ratio of $\mathrm{BH}_{4}$ and total biopterin expression in ihMC lysate. (c) Percentage of $\mathrm{BH}_{4}$ to $\mathrm{BH}_{2}$ oxidation in ihMC lysate. The data was represented as means \pm SD from three independent experiments. $* \mathrm{P}<0.05 ; * * \mathrm{P}<0.01 ; * * * \mathrm{P}<0.001$

Figure 1 
a

\begin{tabular}{|c|c|c|}
\hline \multirow{2}{*}{ Groups } & \multicolumn{2}{|c|}{ Glycaemia (mmol/L) } \\
\hline & Initial & Final \\
\hline Normal & $8.6 \pm 0.4$ & $8.4 \pm 0.5$ \\
\hline $\mathrm{DM}$ & $18.5 \pm 2.3 * * *$ & $17.3 \pm 2.2 * * *$ \\
\hline DM+DJC & $17.3 \pm 1.9 * * *$ & $8.0 \pm 0.8 \#$ \\
\hline DM+Leeches & $18.4 \pm 2.0 * * *$ & $11.3 \pm 1.2 \#$ \\
\hline $\mathrm{DM}+\mathrm{PP}$ & $17.0 \pm 2.9 * * *$ & $12.4 \pm 1.9 *$ \\
\hline $\mathrm{DM}+\mathrm{PSA}$ & $18.3 \pm 2.5 * \star *$ & $10.9 \pm 1.4 \#$ \\
\hline $\mathrm{DM}+\mathrm{RGL}$ & $18.6 \pm 2.0 * * *$ & $11.2 \pm 1.5^{\#}$ \\
\hline
\end{tabular}

b

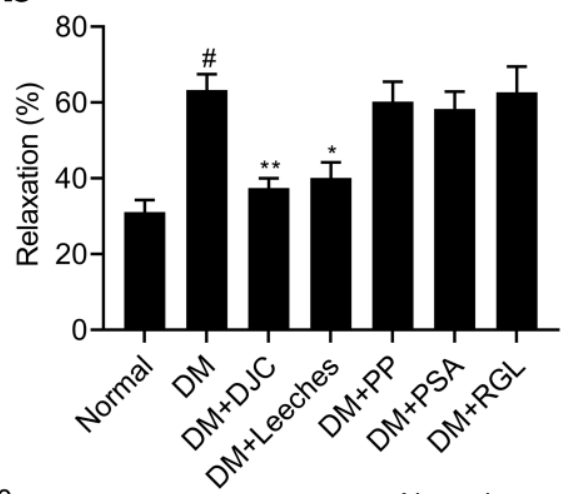

C

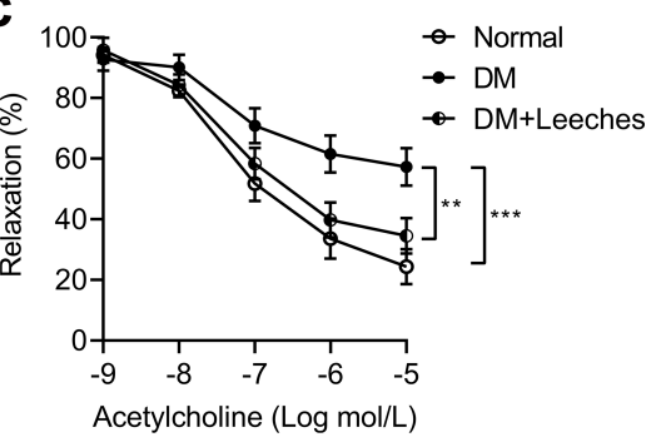

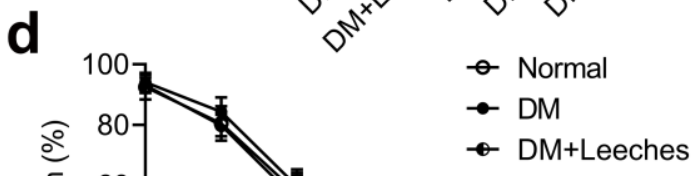


Figure 2

a

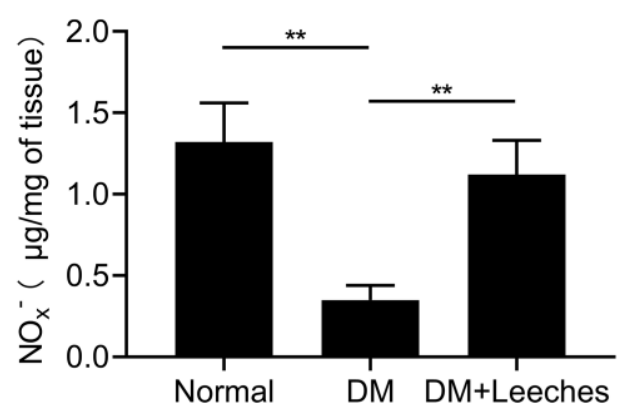

C

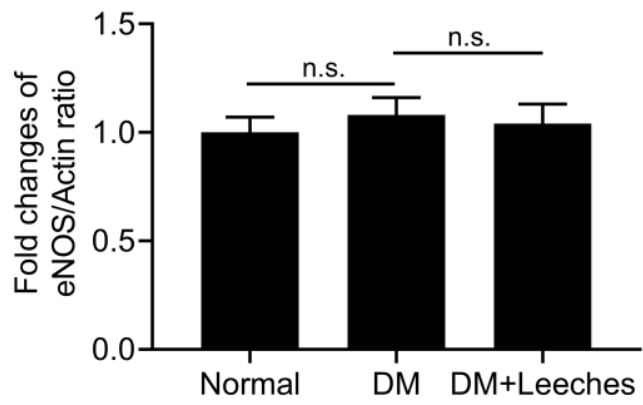

b

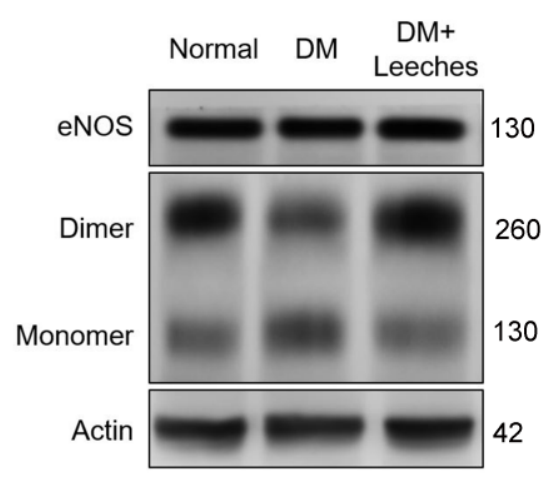

d

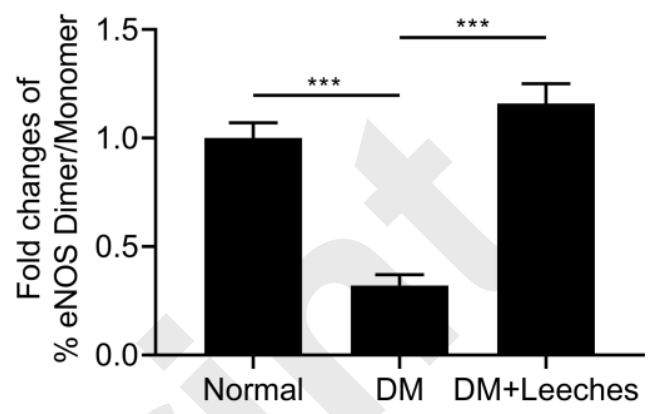


Figure 3
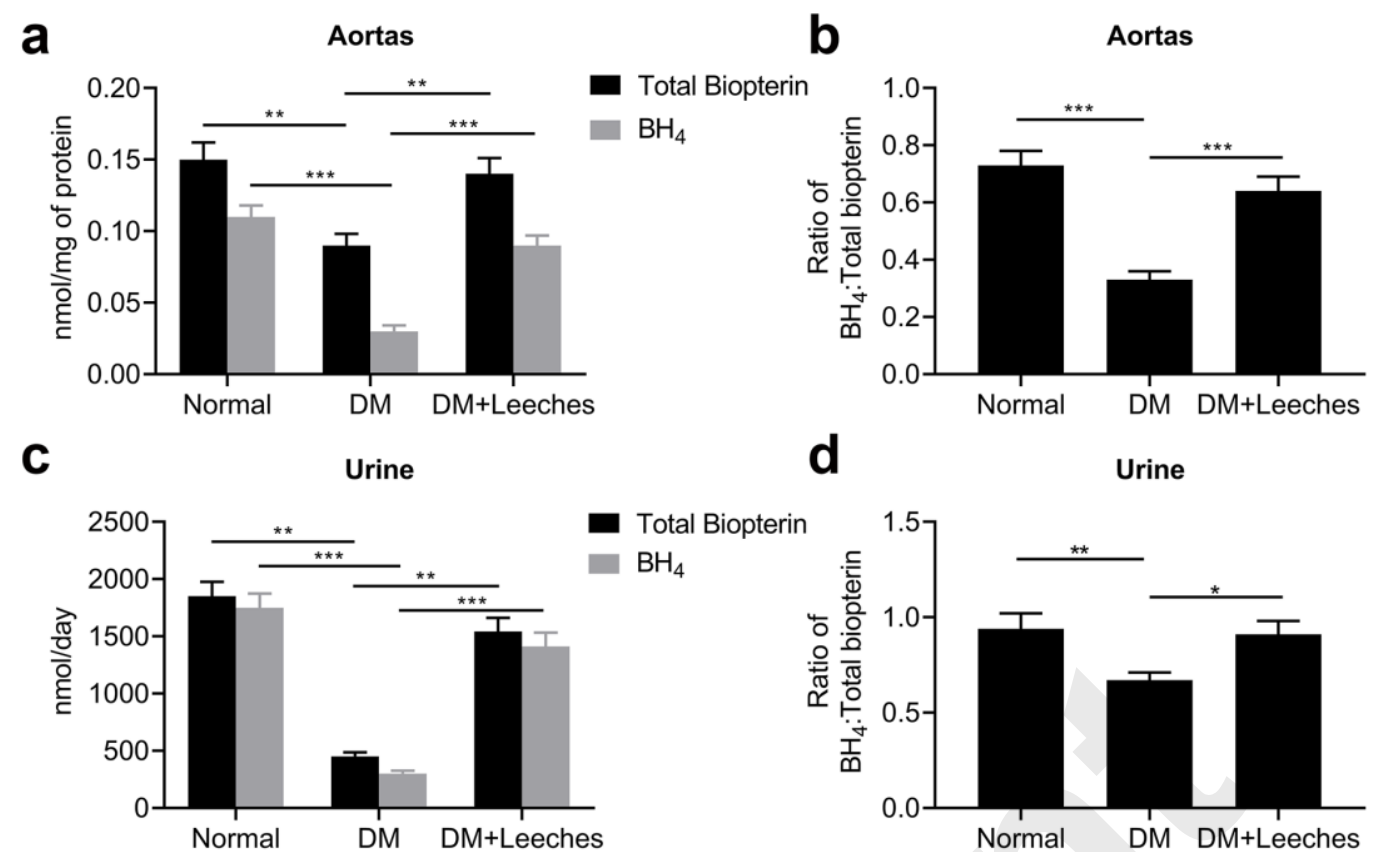

e

Aortas

f

Urine
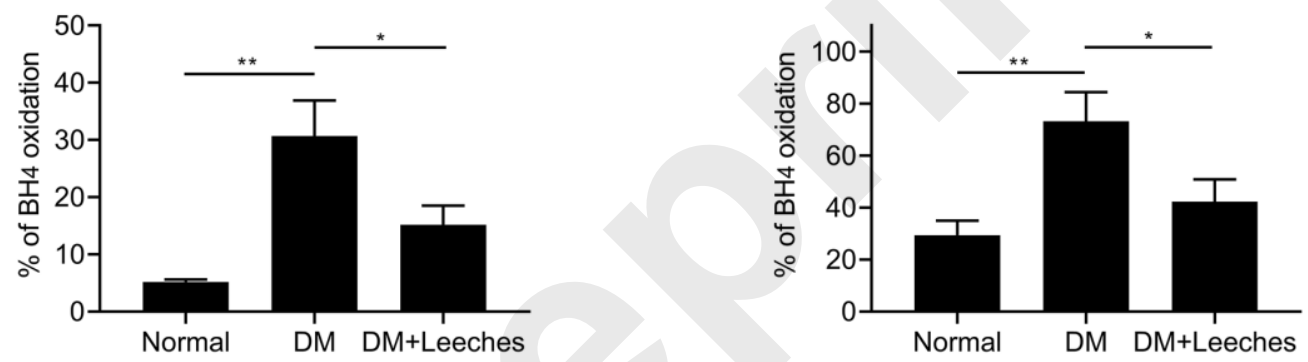
Figure 4

a
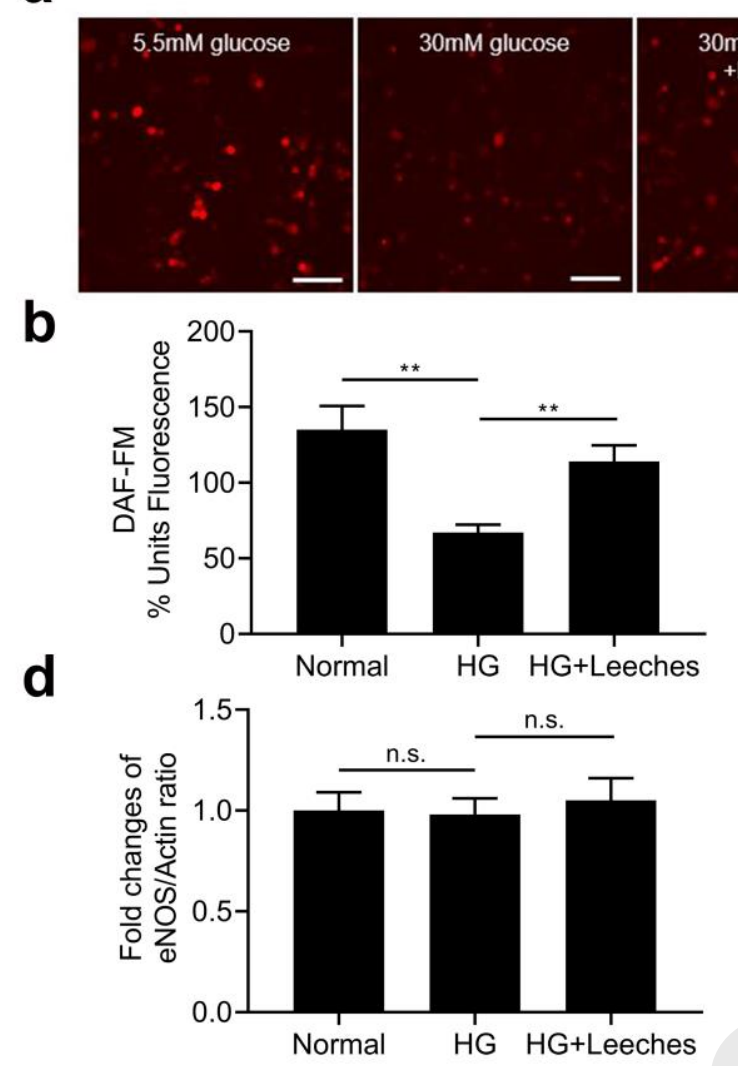

C

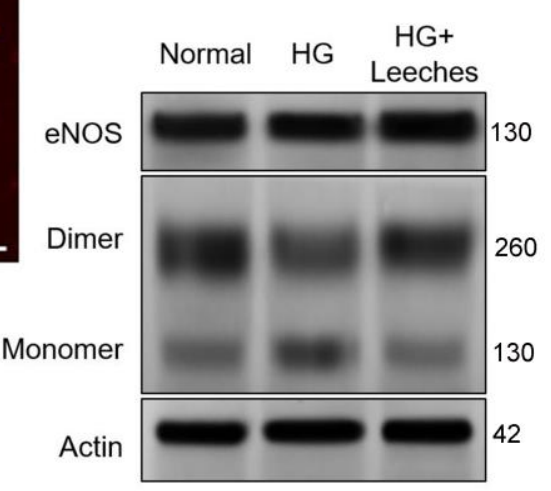

e

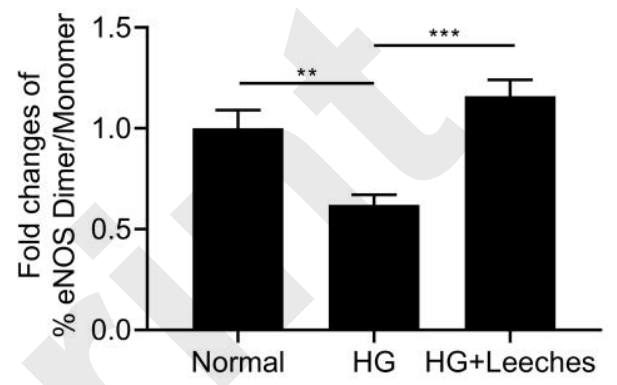


Figure 5

a

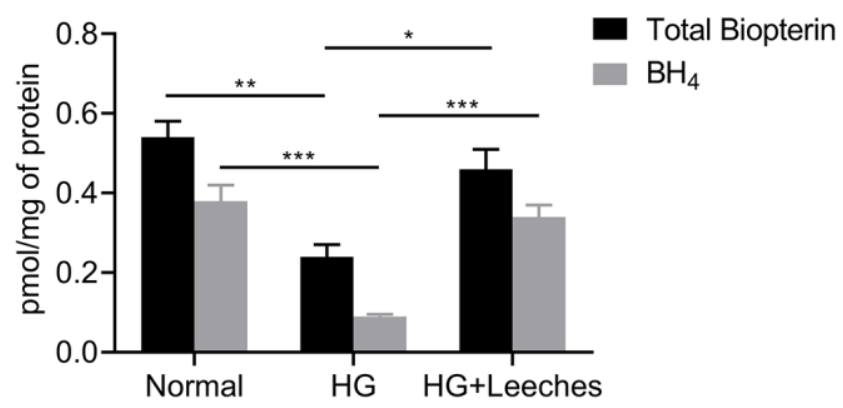

b

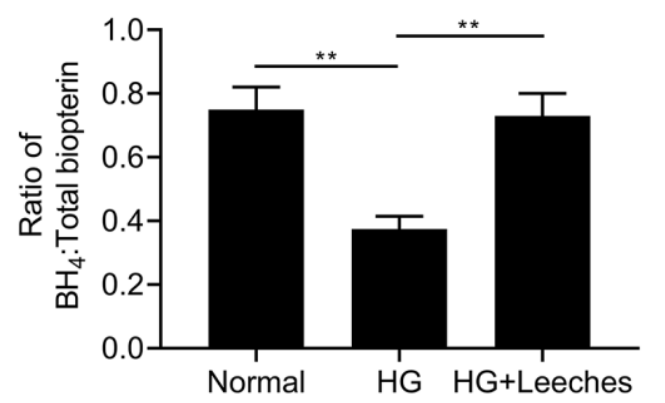

C

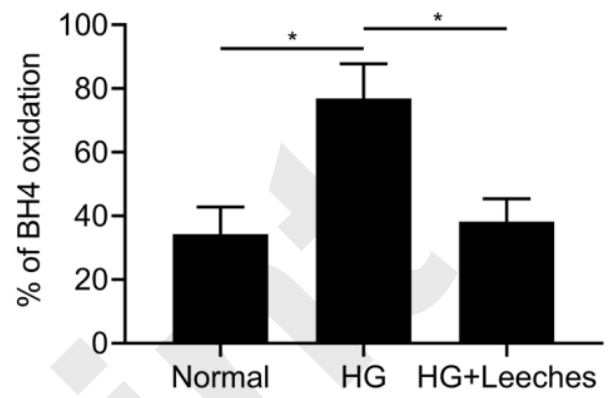

